\section{Evolução e classificação do peso corporal em relação aos resultados da cirurgia bariátrica - derivação gástrica em Y de Roux}

\author{
Body weight evolution and classification of body weight in relation \\ to the results of bariatric surgery - Roux-en-Y gastric bypass
}

Patrícia Fátima Sousa Novais ${ }^{1,2}$, Irineu Rasera Junior ${ }^{2,3}$, Celso

Vieira de Souza Leite ${ }^{3}$, Maria Rita Marques de Oliveira ${ }^{1,4}$

\section{RESUMO}

Objetivo: Avaliar a evolução e a classificação do peso corporal em relação aos resultados da cirurgia bariátrica em mulheres submetidas ao procedimento cirúrgico há mais de dois anos. Sujeitos e método: Foram avaliadas 141 mulheres submetidas à derivação gástrica em $Y$ de Roux (DGYR) com anel de contenção. As participantes foram divididas de acordo com o tempo de pós-operatório e conforme o percentual da perda do excesso de peso (\%PEP): $<50 ; 50-1$ 75; e, $\geq 75$. Resulltados: As mulheres do grupo com \%PEP $\leq 50$ (15,6\%) se mantiveram obesas, enquanto aquelas que apresentaram \%PEP $\geq 75(36,2 \%)$ situaram-se entre a eutrofia e préobesidade e tiveram menor índice de recuperação tardia de peso em relação aos demais grupos. Conclusão: A evolução de peso após dois ou mais anos da cirurgia mostrou sua esperada redução com variados graus de resposta, apontando a necessidade de monitoramento, investigação e intervenção para obtenção dos resultados esperados. Arq Bras Endocrinol Metab. 2010;54(3):303-10

Descritores

Obesidade mórbida; cirurgia bariátrica; peso corporal; perda de peso

\begin{abstract}
Objective: The objective of this study was to assess the evolution and classification of body weight in relation to the results of bariatric surgery in women who underwent the procedure more than two years ago. Subjects and method: A total of 141 women underwent banded Roux-en-Y gastric bypass (RYGB). The participants were divided according to the time elapsed since surgery and the percentage of excess weight lost (\%EWL): $<50 ; 50-75$; and $\geq 75$. Results: The women in the group with $\% E W L \leq 50$ (15.6\%) remained obese, while those with $\% E W L \geq 75$ $(36.2 \%)$ ranged from normal to pre-obese and presented lower late weight gain than the women in the other groups. Conclusion: Weight evolution two or more years after surgery showed the expected reductions, with some individuals responding better to surgery than others. This shows that it is necessary to monitor, investigate and intervene to obtain the desired results. Arq Bras Endocrinol Metab. 2010;54(3):303-10
\end{abstract}

Keywords

Morbid obesity; bariatric surgery; body weight; weight loss
1 Programa de Pós-Graduação de Alimentos e Nutrição, Ciências Nutricionais, Universidade Estadual Paulista (Unesp), Faculdade de Ciências Farmacêuticas (UnespFcFar), Araraquara, SP, Brasil ${ }^{2}$ Centro de Gastroenterologia e Cirurgia da Obesidade, Clínica Bariátrica, Hospital Fornecedores de Cana, Piracicaba, SP, Brasil ${ }^{3}$ Faculdade de Medicina, Unesp, Botucatu, SP, Brasil ${ }^{4}$ Instituto de Biociências, Unesp, Botucatu, SP, Brasil
Correspondência para: Maria Rita Marques de Oliveira Universidade Estadual Paulista, Instituto de Biociências, Curso de Nutrição

Distrito de Rubião Junior, s/n, caixa-postal 510

18618-000 - Botucatu, SP, Brasil mrmolive@ibb.unesp.br

Recebido em 16/Maio/2009 Aceito em 7/Dez/2009

\section{INTRODUÇÃO}

$\mathrm{A}_{\mathrm{r}}^{\mathrm{c}}$ obesidade tornou-se epidemia e problema de saúde pública em todo o mundo, sem distinção de sexo e idade (1-3). As consequências da obesidade no indivíduo, principalmente em seu grau mais avançado, são evidentes, como a redução na expectativa e na qualidade de vida, os problemas psicossociais e a incidência de doenças concomitantes (4-6).

Em situação grave, a cirurgia bariátrica tem configurado como o único tratamento para alcançar perda 
de peso adequada e durável (7-9). No Brasil, a cirurgia inclui-se entre os procedimentos de alta complexidade cobertos pelo Sistema Único de Saúde (SUS), conforme regulamentação legal estabelecida em 2001 (10). Já as diretrizes para sua indicação e os cuidados profissionais foram revisados em 2006, no primeiro Consenso Brasileiro Multissocietário em Cirurgia da Obesidade, coordenado pela Sociedade Brasileira de Cirurgia Bariátrica e Metabólica (11). Como se vê, o procedimento é relativamente recente no país e os seus resultados necessitam de constante avaliação.

Após o procedimento bariátrico, a redução do peso é claramente visível, com consequente melhora das comorbidades e da qualidade de vida (9). Uma perda de peso que se traduz como sucesso cirúrgico deve atingir ao menos $50 \%$ do peso excedente no momento da cirurgia (12-16). Alguns autores estabelecem classificação para sucesso de pós-operatório de acordo com os resultados sobre o índice de massa corporal (IMC), sendo o IMC $<30 \mathrm{~kg} / \mathrm{m}^{2}$ considerado excelente resultado, entre 30 e $35 \mathrm{~kg} / \mathrm{m}^{2}$, bom resultado e $>35 \mathrm{~kg} / \mathrm{m}^{2}$, falha ou insucesso (17-19).

A cirurgia não promove cura da obesidade, mas sim controle, e pode estar associada a complicações em diversos momentos (20-21). Se de um lado a restrição energética é acompanhada do risco de aversões alimentares e consumo inadequado de alimentos, que podem levar a desnutrição e carências nutricionais específicas (21), de outro lado, um aumento no consumo energético pode levar à recuperação do peso em longo prazo após a cirurgia, o que deve ser alvo de estudo e monitoramento, com vistas à manutenção da perda de peso obtida com a cirurgia, assim como aos benefícios a ela associados.

Dessa forma, o presente estudo teve como objetivo avaliar a evolução e a classificação do peso corporal em relação aos resultados da cirurgia bariátrica em mulheres submetidas a esse procedimento há mais de dois anos.

\section{SUJEITOS E MÉTODOS}

\section{Casuística}

Participaram do estudo 141 mulheres operadas há mais de dois anos na Clínica Bariátrica - Hospital dos Fornecedores de Cana de Piracicaba - São Paulo, que, além de serviço privado, realiza grande número de cirurgias bariátricas pelo SUS.
Para a inclusão na pesquisa, foram obedecidos os seguintes critérios: idade superior a 21 anos, submissão à cirurgia bariátrica pela técnica de derivação gástrica em Y de Roux (DGYR) por laparotomia ou laparoscopia, ambas com anel de contenção, há pelo menos dois anos, e frequentar o serviço em intervalo mínimo de um ano. Foram incluídas na pesquisa as mulheres que concordaram em participar do estudo, após assinatura do Termo de Consentimento Livre e Esclarecido. Para homogeneização da amostra, foram excluídos da pesquisa 13 homens que responderam ao convite. O estudo foi aprovado pelo Comitê de Ética em Pesquisa (CEP/FCF/CAr n ${ }^{\circ}$ 16/2006).

As mulheres foram recrutadas por meio de contato telefônico, entre os 1.500 indivíduos que haviam realizado a cirurgia no período de 1998 a 2004. O recrutamento foi realizado por ordem aleatória na lista dos indivíduos que atendiam os critérios de inclusão. Tratou-se de uma amostra de conveniência, referente ao número de indivíduos encontrados no momento da ligação, ou que retornaram a ligação aceitando participar do estudo.

\section{Histórico do peso}

Para analisar o histórico do peso, foi feita uma pesquisa nos prontuários eletrônicos do local de estudo. Foram coletados dados do peso corporal no dia da cirurgia, após seis meses, a cada ano completado do procedimento cirúrgico, considerando-se uma margem de tolerância de mais ou menos um mês. Foram ainda coletadas dos prontuários as informações sobre idade, cor da pele, estado civil, técnica cirúrgica (DGRY laparotomia ou DGRY videolaparoscopia).

\section{Avaliação antropométrica}

As medidas antropométricas atuais de peso foram obtidas em visita das voluntárias à clínica, especialmente para os fins da pesquisa. A aferição dessas medidas seguiu o proposto por Gibson (22). Foram analisados:

Índice de massa corporal (IMC, $\mathrm{em} \mathrm{kg} / \mathrm{m}^{2}$ ): obtido do peso corporal em quilos dividido pela altura em metros ao quadrado.

Excesso de peso na cirurgia (EP, em kg): diferença do peso pré-cirurgia em relação ao peso ideal (23).

Peso perdido (PP, em $\mathrm{kg})$ : diferença do peso précirurgia em relação ao atual.

Perda percentual do excesso de peso (\%PEP): diferença percentual do peso perdido em relação ao excesso de 
peso, que foi empregada como indicador de sucesso da cirurgia $(9,14,24)$.

Peso recuperado (PR, em kg): diferença do peso atual em relação ao menor peso obtido após a cirurgia.

Percentual de peso recuperado (\%PR): diferença percentual do peso atual em relação ao menor peso obtido após a cirurgia.

\section{Análise dos dados}

Para análise dos dados referentes à caracterização da população, as participantes da pesquisa foram divididas em grupos de acordo com o \%PEP: $<50 ; 50 \dashv 75$ e $\geq 75$. A análise retrospectiva da evolução do peso corporal foi realizada de duas maneiras: conforme os períodos pós-operatórios de 6 meses, 1 ano, 2 anos, 3 anos, 4 anos e mais de 5 anos após a cirurgia, considerando o grupo no seu todo, ou com as mulheres distribuídas em grupos conforme o tempo de cirurgia, ano a ano, a partir de 2 até 5 ou mais anos.

Os dados foram tabulados com auxílio do software Excel $^{\circledR}$ e a análise estatística, com auxílio do programa BioEstat $3^{\circledast}(25)$. Todas as variáveis contínuas registradas foram tabeladas como média \pm desvio-padrão ou mediana acompanhada dos valores máximos e mínimos. As variáveis nominais foram expressas em percentagem.

Entre as variáveis contínuas, antes da aplicação do teste estatístico todos os conjuntos de dados foram testados para a normalidade. As comparações entre mais de dois conjuntos de dados foram testadas pela análise de variância (ANOVA), seguida do teste de Tukey para comparações entre as médias. Para os dados não paramétricos, foi utilizado o teste Kruskal-Wallis, seguido do teste de Dunn para comparações entre as medianas. A comparação entre duas medianas foi realizada pelo teste de Mann Whitney.

As comparações nas distribuições proporcionais das variáveis entre os grupos foram realizadas pelo teste qui-quadrado, agrupando-se os valores nulos, bem como os valores esperados menores que 5 . A significância estatística considerada foi de $5 \%(\mathrm{p} \leq 0,05)$ em todas as operações efetuadas.

\section{RESULTADOS}

A caracterização das participantes segundo o \%PEP é mostrada na tabela 1. Pelo critério utilizado para classificação do sucesso da cirurgia (\%PEP $\geq 50)$, foi verificado que $84 \%$ das mulheres obtiveram o sucesso esperado.
Tabela 1. Caracterização das participantes da pesquisa distribuídas conforme a perda percentual do excesso de peso (\%PEP)

\begin{tabular}{|c|c|c|c|c|}
\hline \multirow[b]{2}{*}{ Variáveis e categorias } & \multicolumn{3}{|c|}{ \%PEP } & \multirow{2}{*}{$\begin{array}{l}\text { Todos } \\
\text { n (\%) }\end{array}$} \\
\hline & $\begin{array}{l}<50 \% \\
n(\%)\end{array}$ & $\begin{array}{c}50-75 \% \\
n(\%)\end{array}$ & $\begin{array}{l}\geq 75 \% \\
\text { n (\%) }\end{array}$ & \\
\hline Distribuiç̧ão & $22(16)$ & $68(48)$ & $51(36)$ & $141(100)$ \\
\hline \multicolumn{5}{|l|}{ Raça } \\
\hline Branca & $20(14)$ & $60(43)$ & $45(32)$ & $125(89)$ \\
\hline Negra & $2(1)$ & $8(6)$ & $6(4)$ & $16(11)$ \\
\hline \multicolumn{5}{|l|}{ Estado civil } \\
\hline Amasiado & $1(1)$ & $2(1)$ & $3(2)$ & $6(4)$ \\
\hline Casado & $16(11)$ & $51(36)$ & $34(24)$ & $101(72)$ \\
\hline Divorciado & $2(1)$ & $3(2)$ & $2(1)$ & $7(5)$ \\
\hline Solteiro & $3(2)$ & $10(7)$ & $10(7)$ & $23(16)$ \\
\hline Viúvo & $0(0)$ & $2(1)$ & $2(1)$ & $4(3)$ \\
\hline \multicolumn{5}{|l|}{ Procedência } \\
\hline Piracicaba & $16(11)$ & $55(39)$ & $38(27)$ & $109(77)$ \\
\hline Região & $6(4)$ & $13(9)$ & $13(9)$ & $32(23)$ \\
\hline \multicolumn{5}{|l|}{ Técnica cirúrgica } \\
\hline DGRY laparotomia & $18(13)$ & $61(43)$ & $47(33)$ & $126(89)$ \\
\hline DGRY videolaparoscopia & $4(3)$ & $7(5)$ & $4(3)$ & $15(11)$ \\
\hline \multicolumn{5}{|l|}{ Convênios } \\
\hline SUS & $10(7)$ & $42(30)$ & $23(16)$ & $75(53)$ \\
\hline $\begin{array}{l}\text { Particular e convênios } \\
\text { privados }\end{array}$ & $12(9)$ & $26(18)$ & $28(20)$ & $66(47)$ \\
\hline Idade atual (anos) & $48 \pm 10^{\mathrm{a}}$ & $\begin{array}{c}44 \pm 9^{b} \\
p<0,01\end{array}$ & $41 \pm 8^{b}$ & $44 \pm 9$ \\
\hline
\end{tabular}

DGRY: Derivação gástrica em Y-de-Roux. SUS: Sistema Único de Saúde. Não houve diferença na distribuição das variáveis entre os grupos pelo teste qui-quadrado.

*: Os valores foram expressos em média \pm desvio-padrão e aqueles indicados com uma mesma letra na mesma linha não diferem entre si pelo teste de Tukey $(p<0,01)$, após ANOVA.

Das 141 mulheres participantes da pesquisa, a maioria foi de cor branca, casada, com idade média de $44 \pm$ 9 anos. Foi notado que o grupo com menor \%PEP era mais velho, com média de $48 \pm 10$ anos (Tabela 1).

A técnica predominante na pesquisa foi a DGYR por laparotomia com anel de contenção $(90 \%)$. Mais da metade das cirurgias $(54 \%)$ foram realizadas pelo SUS (Tabela 1).

Na tabela 2, são apresentados os resultados referentes às variáveis antropométricas. As participantes da pesquisa no período pré-cirurgia apresentavam indicadores antropométricos semelhantes, quando divididas nos três grupos de PEP\% (Tabela 2).

No quesito histórico do peso, houve diferença estatística entre os grupos em relação ao peso e ao IMC, com valores inversamente proporcionais a $\% \mathrm{PEP}$ (Tabela 2 ). 
Tabela 2. Variáveis antropométricas das participantes da pesquisa distribuídas conforme a percentagem de perda do excesso de peso (\% PEP)

\begin{tabular}{|c|c|c|c|c|c|}
\hline \multirow{3}{*}{ Variáveis } & \multicolumn{3}{|c|}{ \%PEP } & \multirow{3}{*}{$\begin{array}{c}\text { Todos } \\
n=141\end{array}$} & \multirow{3}{*}{$\begin{array}{c}\text { ANOVA } \\
\mathbf{p}\end{array}$} \\
\hline & $<50$ & $50 \nmid 75$ & $\geq 75$ & & \\
\hline & $\mathrm{n}=\mathbf{2 2}$ & $n=68$ & $\mathrm{n}=51$ & & \\
\hline \multicolumn{6}{|l|}{ Pré-cirurgia } \\
\hline Estatura (m) & $1,6 \pm 0,1$ & $1,6 \pm 0,1$ & $1,6 \pm 0,1$ & $1,6 \pm 0,1$ & 0,31 \\
\hline Peso cirurgia (kg) & $116,3 \pm 16,0$ & $120,3 \pm 16,6$ & $113,2 \pm 15,9$ & $117,1 \pm 16,4$ & 0,06 \\
\hline IMC cirurgia $\left(\mathrm{kg} / \mathrm{m}^{2}\right)$ & $45,0 \pm 5,6$ & $46,9 \pm 6,6$ & $45,2 \pm 7,3$ & $45,9 \pm 16,4$ & 0,29 \\
\hline Excesso de peso $(\mathrm{kg})$ & $57,9 \pm 14,7$ & $61,9 \pm 15,8$ & $55,8 \pm 15,9$ & $59,1 \pm 15,9$ & 0,10 \\
\hline Peso ideal* ${ }^{\star}$ & $58,5 \pm 3,1$ & $58,4 \pm 3,6$ & $57,4 \pm 3,3$ & $58,0 \pm 11,6$ & 0,25 \\
\hline \multicolumn{6}{|l|}{ Históricas } \\
\hline Menor peso (kg) & $83,1 \pm 9,1^{\mathrm{a}}$ & $72,2 \pm 9,2^{b}$ & $61,5 \pm 7,2^{c}$ & $69,9 \pm 11,3$ & $<0,05$ \\
\hline Menor IMC (kg/m²) & $32,2 \pm 3,7^{\mathrm{a}}$ & $28,2 \pm 3,6^{b}$ & $24,5 \pm 2,0^{c}$ & $27,5 \pm 4,3$ & $<0,05$ \\
\hline Maior peso (kg) & $91,7 \pm 10,1^{\mathrm{a}}$ & $81,8 \pm 10,1^{b}$ & $66,0 \pm 9,6^{c}$ & $77,5 \pm 13,8$ & $<0,05$ \\
\hline Maior IMC (kg/m²) & $35,4 \pm 3,2^{\mathrm{a}}$ & $31,9 \pm 3,8^{b}$ & $26,1 \pm 3,8^{c}$ & $30,4 \pm 5,0$ & $<0,05$ \\
\hline \multicolumn{6}{|l|}{ Atuais } \\
\hline Peso atual (kg) & $92,0 \pm 10,1^{\mathrm{a}}$ & $80,5 \pm 8,1^{b}$ & $65,1 \pm 6,6^{c}$ & $76,5 \pm 12,2$ & $<0,05$ \\
\hline IMC atual $\left(\mathrm{kg} / \mathrm{m}^{2}\right)$ & $35,5 \pm 3,2^{\mathrm{a}}$ & $31,4 \pm 3,2^{b}$ & $25,9 \pm 2,8^{c}$ & $30,0 \pm 4,5$ & $<0,05$ \\
\hline Recuperação (\%) & $28,8 \pm 1,1^{\mathrm{a}}$ & $10,3 \pm 6,8^{\mathrm{a}}$ & $5,9 \pm 5,0^{b}$ & $8,5 \pm 6,3$ & $<0,05$ \\
\hline$\% \mathrm{PEP}^{\star *}$ & $45,3(32,4-49,3)^{\mathrm{a}}$ & $64,3(50,1-74,8)^{\mathrm{b}}$ & $86,8(75,7-121,3)^{c}$ & $68,5(32,3-121,3)$ & $<0,05$ \\
\hline
\end{tabular}

IMC: índice de massa corporal $\left(\mathrm{kg} / \mathrm{m}^{2}\right)$.

* Metropolitan height and weight tables, 1983. Os valores indicados com uma mesma letra numa mesma linha não diferem entre si pelo teste de Tukey, após ANOVA $(p<0,05)$.

** Dados expressos em mediana (máximo e mínimo), sendo que os valores indicados com uma mesma letra numa mesma linha não diferem entre si pelo teste de Dunn após Kruskal Wallis $(p<0,05)$.

Entre as variáveis do peso atual, o maior peso $(92,0$ $\pm 10,1)$ e o maior IMC $(35,4 \pm 3,2)$ foram encontrados no grupo $<50 \%$ PEP (Tabela 2 ). A recuperação de peso menos expressiva ocorreu no grupo $\geq 75 \%$ PEP com $5,9 \%$ de recuperação de peso (Tabela 2 ).

A tabela 3 descreve a evolução das variáveis da massa corporal conforme o tempo de cirurgia. A evolução nos dois primeiros anos foi referente à totalidade da amostra do estudo. $\mathrm{O}$ menor peso ocorreu entre o primeiro e o terceiro ano após a cirurgia. A partir do quarto $(n=73)$ e do quinto ano $(n=67)$, o teste de Dunn não acusou diferenças no peso e no IMC, respectivamente, em relação ao peso obtido aos seis meses de cirurgia.

A figura 1 apresenta a evolução da perda de peso em quilos entre as mulheres divididas em grupos conforme o tempo de cirurgia, evidenciando a maior perda de peso até o sexto mês e sua estabilização entre o primeiro e o segundo ano da cirurgia.

Na tabela 4 são apresentados os valores da evolução da perda de peso total $(\mathrm{kg})$ entre as mulheres agrupadas conforme o tempo de cirurgia e os períodos de pósoperatórios $(\mathrm{PO})$.
Tabela 3. Variáveis da massa corporal entre as participantes da pesquisa distribuídas conforme 0 tempo de cirurgia bariátrica

\begin{tabular}{lccc}
\hline Variáveis & $\mathbf{n}$ & $\begin{array}{c}\text { Peso }(\mathbf{k g}) \\
\text { MED (MíN-MÁX) }\end{array}$ & $\begin{array}{c}\text { IMC (kg/m²) } \\
\text { MED (MíN-MÁX) }\end{array}$ \\
\hline Pré-operatório & 141 & $115,0(72,3-164,0)^{\mathrm{a}}$ & $45,0(33,0-69,1)^{\mathrm{a}}$ \\
6 meses & 141 & $84,0(53,4-120,0)^{\mathrm{b}}$ & $32,5(21,3-50,5)^{\mathrm{b}}$ \\
1 ano & 141 & $74,6(49,8-109,2)^{\mathrm{c}}$ & $29,3(20,5-45,0)^{\mathrm{c}}$ \\
2 anos & 141 & $72,6(52,0-107,0)^{\mathrm{c}}$ & $28,3(20,3-44,0)^{\mathrm{c}}$ \\
3 anos & 111 & $74,6(56,6-106,0)^{\mathrm{c}}$ & $29,1(21,8-43,6)^{\mathrm{c}}$ \\
4 anos & 73 & $79,5(57,2-108,0)^{\mathrm{bc}}$ & $30,1(21,3-44,4)^{\mathrm{c}}$ \\
$\geq 5$ anos & 67 & $79,0(55,0-100,6)^{\mathrm{bc}}$ & $31,0(22,6-37,6)^{b c}$ \\
& & $p<0,0000^{*}$ & $p<0,0000^{*}$ \\
\hline
\end{tabular}

IMC: índice de massa corporal; MED: mediana; MíN: mínimo; MÁX: máximo.

*: teste de Kruskal Wallis; os valores indicados com uma mesma letra numa mesma coluna não diferem entre si pelo teste de Dunn $(p<0,05)$.

Entre os grupos estudados, as mulheres operadas há cinco anos ou mais apresentaram maior peso no período pré-cirurgia. Quando completados seis meses de PO, houve diferença estatística entre os grupos que realizaram o procedimento cirúrgico havia três anos e quatro anos, cujo peso foi mais elevado (Tabela 4). 
Tabela 4. Evolução da perda de peso $(\mathrm{kg})$ entre as participantes da pesquisa agrupadas por tempo da cirurgia e período de pós-operatório

\begin{tabular}{lccccc}
\hline Variáveis & $\begin{array}{c}\text { G2a }(\mathbf{n}=\mathbf{3 0}) \\
\text { MED (MíN-MÁX) }\end{array}$ & $\begin{array}{c}\text { G3a }(\mathbf{n}=\mathbf{3 8}) \\
\text { MED (MíN-MÁX)) }\end{array}$ & $\begin{array}{c}\text { G4a }(\mathbf{n}=\mathbf{3 3}) \\
\text { MED (MíN-MÁX)) }\end{array}$ & $\begin{array}{c}\mathbf{G} \geq \mathbf{5 a}(\mathbf{n}=\mathbf{4 0}) \\
\text { MED (MíN-MÁX) }\end{array}$ & Intergrupos \\
\hline Pré-operatório & $114(72-145)^{\mathrm{a}, \mathrm{A}}$ & $109(86-148)^{\mathrm{a}, \mathrm{A}}$ & $115(97-161)^{\mathrm{a}, \mathrm{A}}$ & $122(91-167)^{\mathrm{b}, \mathrm{A}}$ & $\mathrm{p}<0,05$ \\
6 meses & $81(53-118)^{\mathrm{ab}, \mathrm{B}}$ & $80(59-112)^{\mathrm{a}, \mathrm{B}}$ & $88(63-120)^{\mathrm{b}, \mathrm{B}}$ & $89(68-118)^{\mathrm{ab}, \mathrm{B}}$ & $\mathrm{p}<0,05$ \\
1 ano & $68(52-98)^{\mathrm{a}, \mathrm{B}}$ & $71(50-96)^{\mathrm{abc}, \mathrm{B}}$ & $83(56-109)^{\mathrm{bc}, \mathrm{B}}$ & $80(57-109)^{\mathrm{c}, \mathrm{C}}$ & $\mathrm{p}<0,05$ \\
2 anos & $66(52-97)^{\mathrm{a}, \mathrm{C}}$ & $71(53-90)^{\mathrm{ab}, \mathrm{B}}$ & $74(58-107)^{\mathrm{ab}, \mathrm{B}}$ & $75(60-102)^{\mathrm{b}, \mathrm{C}}$ & $\mathrm{p}<0,05$ \\
3 anos & - & $73(57-92)^{\mathrm{B}}$ & $78(59-106)^{\mathrm{B}}$ & $75(60-96)^{\mathrm{C}}$ & $\mathrm{p}<0,05$ \\
4 anos & - & - & $80(57-108)^{\mathrm{B}}$ & $77(63-100)^{\mathrm{C}}$ & $\mathrm{p}=0,57^{\star}$ \\
Intergrupo & $\mathrm{p}<0,05$ & $\mathrm{p}<0,05$ & $\mathrm{p}<0,05$ & $\mathrm{p}<0,05$ & \\
\hline
\end{tabular}

G2a: 2 anos da cirurgia; G3a: 3 anos da cirurgia; G4a: 4 anos da cirurgia, $G \geq 5^{\text {a: }} 5$ ou mais anos da cirurgia. MED: mediana; MíN: mínimo; MÁX: máximo.

Os valores indicados com uma mesma letra minúscula numa mesma linha não diferem entre si pelo teste de Dunn após o teste de Kruskal-Wallis, enquanto os valores indicados com uma mesma letra maiúscula numa mesma coluna não diferem entre si pelo teste de Dunn após o teste de Kruskal-Wallis.

* Teste de Mann Whitney.

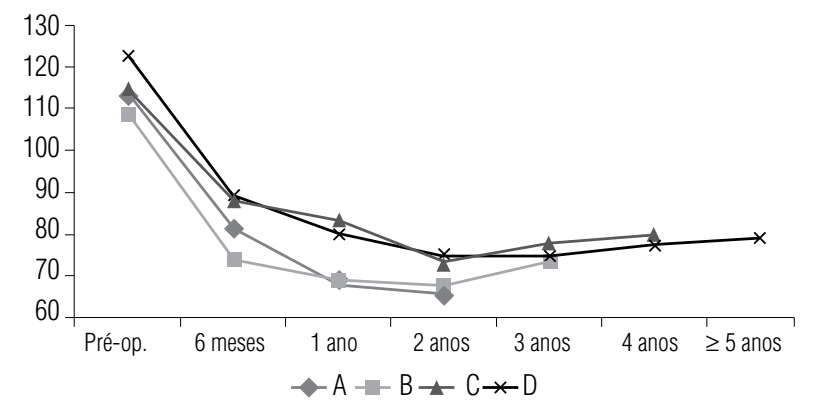

Pré-op: peso pré-operatório; A: 2 anos; B: 3 anos; C: 4 anos; D: $\geq 5$ anos

Figura 1. Evolução do peso corporal entre as participantes da pesquisa distribuídas conforme 0 tempo (em anos) da cirurgia bariátrica. Dados expressos em mediana de peso corporal, em kg.

No primeiro ano de $\mathrm{PO}$, a diferença ocorreu entre as operadas há dois anos em relação às operadas há quatro anos e àquelas com mais de cinco anos, as quais apresentaram maiores valores de peso (Tabela 4).

Quando completados dois anos da cirurgia, observou-se que as mulheres operadas há dois anos apresentaram menor peso em relação às operadas há mais de cinco anos. Nos outros momentos de pós-operatório, não foram encontradas diferenças entre os grupos de acordo com o período em que foi realizada a cirurgia (Tabela 4).

Nas comparações intragrupos (Tabela 4), confirmou-se que a maior perda de peso ocorre até o sexto mês, e as reduções posteriores não foram sensíveis aos testes estatísticos nos grupos três e quatro anos de cirurgia. No grupo dois anos, a redução de peso mostrou-se significativa também aos dois anos da cirurgia, enquanto no grupo com mais de cinco anos a redução foi significativa em um ano da cirurgia.

\section{DISCUSSÃO}

A perda de peso é considerada um dos principais parâmetros para definir o sucesso da cirurgia bariátrica, já que, após o emagrecimento, ocorre comprovada melhora nas condições clínicas do indivíduo (20,26-29). É consenso predominante entre investigadores que o critério para avaliação do sucesso no tratamento cirúrgico da obesidade é a \%PEP de pelo menos $50 \%$ e a manutenção de peso em longo prazo (13-16,24). A partir desse critério, as participantes do presente estudo foram divididas e analisadas em três grupos de \%PEP: $<50 \%$; $50-75 \% ; \geq 75 \%$, sendo o primeiro grupo caracterizado como insucesso e os dois outros grupos, como sucesso em relação à perda de peso (13-16,24).

A perda de peso está diretamente relacionada à significativa melhora do estado metabólico e, consequentemente, à redução de riscos associados à obesidade (20,26-29). O mecanismo da perda de peso após a cirurgia envolve fatores mecânicos e hormonais. Inicialmente, pode-se citar a redução na ingestão de energia em decorrência da menor capacidade gástrica. Já a provável modificação hormonal que ocorre após o procedimento cirúrgico, ocasionando a redução de apetite, pode ser gerada pela diminuição da grelina sérica e aumento do peptídeo Y e glucagon-like peptídeo 1 (GLP-1) (30).

A forma como o conjunto de fatores envolvidos no emagrecimento pós-cirúrgico interage é que determina os resultados da cirurgia sobre o peso corporal, em curto e em longo prazo. Daí a importância de se conhecer 
o padrão de emagrecimento e os fatores a ele relacionados. Há também que se levar em conta o monitoramento dos resultados da cirurgia, especialmente em longo prazo, para que se comprove a manutenção dos benefícios do procedimento.

Neste estudo, a idade foi um fator associado negativamente ao sucesso da cirurgia e não o peso pré-cirúrgico. As mulheres mais velhas independentemente do peso corporal responderam com menor \%PEP, o que já foi documentado em outros estudos $(31,32)$.

A média do \% PEP $(68,5 \%)$ encontrada neste estudo está de acordo com o que é apresentado na literatura para a DGYR ou a técnica correspondente. Em metaanálise realizada por Buchwald e cols. (33), com 10.172 indivíduos submetidos ao procedimento cirúrgico, foi encontrada \%PEP média de $61,2 \%$ após dois anos de seguimento. Resultado semelhante foi encontrado no estudo realizado por Campos e cols. (34), que apresentaram \%PEP de 60,2\% entre 310 indivíduos com seguimento de 12 meses de PO. Awad e cols. (35), após estudarem 244 indivíduos obesos operados, verificaram que depois de 6 meses havia uma \%PEP de 55\%, após um ano de $67,8 \%$ e após dois anos de $73 \%$. No estudo de Wolf e Beisiegel (36), os pacientes atingiram \% PEP de $50 \%$ após sete meses de cirurgia. Esses valores se repetem em outros dois estudos que apresentam \% entre $50 \%$ e $77 \%$ após um ano de cirurgia $(37,38)$.

Nos dados antropométricos (peso e IMC) referentes ao período pré-operatório, foram observados valores semelhantes entre os grupos divididos conforme a \%PEP, o que concorda com outros estudos, nos quais o peso da cirurgia não foi fator determinante do seu resultado (7). Já nos dados antropométricos históricos, foi confirmada a diferença entre todos os grupos, sendo observado que as mulheres que apresentaram menores \%PEP se mantiveram obesas em relação à classificação do IMC (obesidade grau 1 no menor peso e obesidade grau 2 no maior peso). As participantes da pesquisa que apresentaram $\%$ PEP entre $50 \nmid 75$ foram classificadas na faixa entre pré-obesidade e obesidade. Naquelas que apresentaram $\% \mathrm{PEP} \geq 75$, a faixa de variação situou-se entre a classificação de eutrofia e pré-obesidade (Tabela 2).

Empregando-se o critério de avaliação do sucesso da cirurgia pelo IMC, no presente estudo confirma-se o insucesso entre o grupo com $\% \mathrm{PEP}$ inferior a $50 \%$, que teve IMC médio de $35,5 \mathrm{~kg} / \mathrm{m}^{2}$, e o bom resultado do grupo com \%PEP de $50 \nmid 75 \%$, com IMC médio de $31,4 \mathrm{~kg} / \mathrm{m}^{2}$. Já o grupo com $\%$ PEP $\geq 75$ teve um excelente resultado com IMC médio de $25,9 \mathrm{~kg} / \mathrm{m}^{2}$.
Quando a análise da evolução do peso foi realizada com as mulheres agrupadas conforme o tempo de pósoperatório, a diferença no peso ocorreu entre seis meses e um ano do pós-operatório. Nessa mesma análise, observa-se que o menor peso ocorreu entre o primeiro e o terceiro ano após a cirurgia (Tabela 3). Resultado semelhante foi observado na meta-análise realizada por Buchwald e cols. (33), na qual não foi notada diferença significativa em relação ao peso na maioria dos estudos a partir do segundo ano de pós-operatório.

Em relação à recuperação do peso, com o decorrer dos anos não houve recuperação significativa. No entanto, quando as mulheres foram divididas nos grupos de estudo conforme a \%PEP, aquelas com $\% \mathrm{PEP} \geq 75 \%$ obtiveram menor índice de recuperação de peso $(5,9 \%) \mathrm{em}$ relação aos demais grupos com menor \%PEP (Tabela 2), determinando que aquelas que emagrecem menos são as que voltam a ganhar peso em grau mais significativo.

Os resultados da cirurgia bariátrica (DGYR) sobre a perda de peso estão comprovados e bem documentados, porém após dois anos do procedimento tem sido observado algum grau de recuperação do peso perdido $(18,39)$. Essa recuperação do peso após a cirurgia, que é considerada o tratamento mais efetivo para o controle da obesidade, comprova o conceito de que obesidade é uma doença crônica, progressiva, que não tem cura e necessita de tratamento específico mesmo após a cirurgia (14).

A recuperação do peso pode ocorrer devido a processos de adaptações fisiológicas no trato gastrointestinal que acontecem com o passar do tempo. A adoção e a promoção de estilo de vida saudável fortalecem o indivíduo operado contra os antigos hábitos que causaram a condição de obesidade. Esse novo comportamento é fundamental para a manutenção em longo prazo do peso alcançado (40).

A evolução anual da \%PEP no pós-operatório (PO), como abordada na presente análise, tem sido pouco explorada na literatura e comumente é estabelecida uma média/mediana do \%PEP para o período estudado. E, quando a evolução do peso é estudada, raramente ultrapassa dois anos do procedimento cirúrgico. O desenho da curva de perda de peso torna-se útil e relevante para o estudo do emagrecimento no decorrer do tempo. No presente estudo, o primeiro semestre caracterizou a fase de perda rápida de peso, seguida de uma fase lenta, que se estendeu entre o primeiro e o segundo ano da cirurgia. A partir do segundo ano, pode-se inferir que houve um sutil indício de recuperação de peso (Tabela 4). A partir da curva de perda de peso, é possível afirmar 
que a perda aos seis meses representa um indicador importante do sucesso da cirurgia.

A perda de peso em quilos no interior dos grupos apresentou diferenças estatisticamente significativas entre o peso da cirurgia e o peso aos seis meses de pósoperatório. Depois disso, as diferenças não se confirmaram pelos testes estatísticos, mostrando que houve estabilização do peso sem recuperação significativa entre os grupos. Houve exceção nos grupos das mulheres com dois e com cinco anos ou mais de cirurgia, que apresentaram diferença significativa na perda de peso também entre seis meses e dois anos e seis meses e um ano após o procedimento cirúrgico, respectivamente (Tabela 4). É possível que o prolongamento da perda de peso entre as participantes que operaram há menos tempo seja justificada pela atuação da equipe multidisciplinar mais bem estruturada nos últimos anos no local do estudo. Já entre as mulheres operadas há cinco anos ou mais, pode-se postular a hipótese de que o maior peso no período antecedente à cirurgia tenha prolongado o tempo de emagrecimento.

Conclui-se que a cirurgia bariátrica pela técnica de DGYR com anel de contenção promove adequada redução do peso corporal, com boa manutenção da perda em médio prazo e variado grau de resposta. O melhor resultado em relação à indesejada recuperação ponderal tardia foi associado às pacientes com maior perda do excesso de peso. Esses resultados confirmam a necessidade de monitoramento, investigação e intervenção para garantir os esperados resultados da cirurgia.

Apoio financeiro para a pesquisa: Fundação de Amparo à Pesquisa do Estado de São Paulo (Fapesp).

Declaração: os autores declaram não haver conflitos de interesse científico neste estudo.

\section{REFERÊNCIAS}

1. Monteiro CA, Conde WL, Popkin BM. Is obesity replacing or adding to undernutrition? Evidence from different social classes in Brazil. Public Health Nutr. 2001;5(1A):105-12.

2. World Health Organization. Obesity and overweight. What are overweight and obesity? September, 2006. Acesso em: 17 de janeiro de 2009. Disponível em: http://www.who.int/mediacentre/ factsheets/fs311/en/print.html

3. James WP. The epidemiology of obesity: the size of the problem. J Intern Med. 2008;263(4):336-52.

4. Wolf AM, Falcone AR, Kortner B, Kuhlmann HW. Baros: an effective system to evaluate the results of patients after bariatric surgery. Obes Surg. 2000;10(5):445-50.

5. Fernandes LC, Pucca $L$, Matos D. Tratamento cirúrgico da obesidade. J Bras Med. 2001;80(3):44-9.

6. O'Brien PE, Brown W, Dixon JB. Obesity, weight loss and bariatric surgery. Med J Aust. 2005;183(6):310-4.
7. Buchwald H. Bariatric surgery for morbid obesity: health implications for patients, health professionals, and third-party payers. Consensus Conference Panel. J Am Coll Surg. 2005;200(4):593-604.

8. Mrad BA, Stoklossa CJ, Birch DW. Does preoperative weight loss predict success following surgery for morbid obesity? Am J Surg. 2008;5:570-4.

9. Steffen R, Potoczna N, Bieri N, Fritz FH. Successful multi-intervention treatment of severe obesity: a 7-year prospective study with 96\% follow-up. Obes Surg. 2009;19:3-12.

10. BRASIL. Portaria $n^{\circ} 628 / G M$ Brasília: Ministério da Saúde/Gabinete do Ministro; 2001.

11. Sociedade Brasileira de Cirurgia Bariátrica, Colégio Brasileiro de Cirurgiões, Colégio Brasileiro de Cirurgia Digestiva, Sociedade Brasileira de Cirurgia Laparoscópica, Associação Brasileira para o Estudo da Obesidade, Sociedade Brasileira de Endocrinologia e Metabologia. Consenso Brasileiro Multissocietário em Cirurgia da Obesidade. 2006. Disponível em: http://www.sbcbm.org.br/ membros_consenso_bariatrico.php. Acesso em: ago, 2009.

12. Brolin RL, Robertson LB, Kenler HA, Cody RP. Weight loss and dietary intake after vertical banded gastroplasty and roux-em-y gastric bypass. Ann Surg. 1994;220(6):782-90.

13. Brolin RE. Bariatric surgery and long-term control of morbid obesity. JAMA. 2002;288:2793-6.

14. Brolin RE. Weight gain after short- and long-limb gastric bypass in patients followed for longer than 10 years. Ann Surg. 2007;246(1):163-4.

15. Fobi MA. Surgical treatment of obesity: a review. J Natl Med Assoc. 2004;96(1):61-75.

16. Silver HJ, Torquati A, Jensen GL, Richards WO. Weight, dietary and physical exercises behaviors two years after gastric bypass. Obes Surg. 2006;16:859-64.

17. Gumbs AA, Pomp A, Gagner M. Revisional bariatric surgery for inadequate weight loss. Obes Surg. 2007;17(9):1137-45.

18. Reinhold RB. Critical analysis of long term weight loss following gastric bypass. Surg Gynecol Obstet. 1982;155:385-94.

19. Christou NV, Look D, Maclean L. Weight gain after short and longlimb gastric bypass in patients followed for longer than 10 years. Ann Surg. 2006;244:734-40.

20. Waitman JA, Aronne LJ. Obesity surgery: pros and cons. J Endocrinol Invest. 2002;25(10):925-8.

21. Malinowski SS. Nutritional and metabolic complications of bariatric surgery. Am J M Sci. 2006;331(4):219-25.

22. Gibson RS. Nutritional assessment: a laboratory manual. New York: Oxford University Press; 1993.

23. Metropolitan height and weight tables. Stat Bul Metrop Live Found. 1983;64(1):3-9.

24. Capella JF, Capella RF. The weight reduction operation of choice: vertical banded gastroplasty or gastric bypass? Amer J Surg. 1996;171:74-9.

25. Ayres M, Ayres Jr M, Ayres DL, Santos AAS. BioEstat aplicações estatísticas nas áreas das ciências bio-médicas, versão 3.0. 2003; Belém: Sociedade Civil Mamirauá/MCT CNPq.

26. Brolin RL, Robertson LB, Kenler HA, Cody RP. Weight loss and dietary intake after vertical banded gastroplasty and Roux-em-Y gastric bypass. Ann Surg. 1994;220(6):782-90.

27. Sjostrom $L$, Lindroos AK, Peltonen $M$, Peltonen $M$, Torgerson J, Bouchard C, et al. Lifestyle, diabetes, and cardiovascular risk factors 10 years after bariatric surgery. $\mathrm{N}$ Engl J Med. 2004;351(26):2683-93.

28. O'Brien PE, Brown W, Dixon JB. Obesity, weight loss and bariatric surgery. Med J Aust. 2005;183(6):310-4.

29. Bult MJF, Van Dalen T, Muller AF. Surgical treatment of obesity. Eur J Endocrinol. 2008;158(2):135-45.

30. Valverde I, Puente J, Martin-duce A, et al. Changes in glucagonlike peptide 1 (GLP-1) secretion after biliopancreatic diversion or vertical banded gastroplasty in obese subjects. Obes Surg. 2005;15:387-97. 
31. Wang $Q$, Hassager $C$, Ravn P, Wang S, Christiansen C. Total and regional body composition changes in early postmenopausal women: age-related or menopause-related? Am Clin Nutr. 1994;60:843-8.

32. De Lorenzi DRS, Basso E, Fagundes PO, Sacilot B. Prevalência de sobrepeso e obesidade no climatério. Rev Bras Ginecol Obstet. 2005;27(8):479-84.

33. Buchwald $H$, Avidor $Y$, Braunwald E, Jensen MD, Pories W, Fahrbach $\mathrm{K}$, et al. Bariatric surgery: a systematic review and metaanalysis. JAMA. 2004;292(14):1724-37.

34. Campos GM, Rabl C, Mulligan K, Posselt A, Rogers SJ, Westphalen $\mathrm{AC}$, et al. Factors associated with weight loss after gastric bypass. Arch Surg. 2008;143(9):877-84.

35. Awad W, Garay A, Martínez C, Onate V, Turu I, Yarmuch J. Descenso ponderal y calidad de vida mediante la cirugía de Bypass gástrico con y sin anillo de calibración. Rev Chil Cir. 2008;60(1):17-21.
36. Wolf AM, Beisiegel U. The effect of loss of excess weight on the metabolic risk factors after bariatric surgery in morbidly and super-obese patients. Ob Surg. 2007;17: 910-9.

37. Schauer PR, Ikramuddin S, Gourash W, Ramanathan R, Luketich J. Outcomes after laparoscopic Roux-en-Y gastric bypass for morbid obesity. Ann Surg. 2000;232:515-29.

38. Higa KD, HoT, Boone KB. Laparoscopic Roux-en-Y gastric bypass: technique and 3-year follow-up. J Laparoendosc Adv Surg Tech A. 2001;11:377-82.

39. Lopez PP, Patel NA, Koche LS. Outpatient complications encountered following Roux-en-Y gastric bypass. Med Clin North Am. 2007;91:471-83.

40. Bond DS, Evans RK, Demaria EJ, Meador RN, Warren BJ, Shannon KA, et al. A conceptual application of health behavior theory in the design and implementation of a successful surgical weight loss program. Obes Surg. 2004;14:849-56. 